\title{
Quasi-deterministic millimeter-wave channel models in MiWEBA
}

\author{
Richard J. Weiler ${ }^{{ }^{*}}$, Michael Peter ${ }^{1}$, Wilhelm Keusgen ${ }^{1}$, Alexander Maltsev ${ }^{2,3}$, Ingolf Karls², Andrey Pudeyev², \\ llya Bolotin ${ }^{2}$, Isabelle Siaud ${ }^{4}$ and Anne-Marie Ulmer-Moll ${ }^{4}$
}

\begin{abstract}
This article introduces a quasi-deterministic channel model and a link level-focused channel model, developed with a focus on millimeter-wave outdoor access channels. Channel measurements in an open square scenario at $60 \mathrm{GHz}$ are introduced as a basis for the development of the model and its parameterization. The modeling approaches are explained, and their specific area of application is investigated.
\end{abstract}

Keywords: Millimeter-wave, Channel model, Access, Backhaul

\section{Introduction}

The increasing mobile traffic demand has led to the proposition of numerous approaches for the future development of mobile radio networks. One popular proposition for the next generation (so-called 5G) is the usage of previously unused spectrum in the millimeterwave band [1]. Using the spectrum at these high frequencies incurs new challenges, compared to radio systems that operate at frequencies below $6 \mathrm{GHz}$. The possible applications are in backhaul and fronthaul links on the network side [2] or on the access link. Recent research proves the general feasibility of outdoor access links in the lower millimeter-wave band $(30-40 \mathrm{GHz})$ based on path loss evaluations [3]. At the same time, a high spatial selectivity of the channel is observed in this publication.

The higher free-space path loss motivates a design shift from an omni-directional operation to more spatial focusing, using high-gain beam-forming antennas and multiple input/multiple output (MIMO) approaches. It will also incur a change in system design, towards a more dense deployment of so-called overlay small cell base stations, which will exist in addition to today's macro-cell deployments. These base stations can then provide very high throughput millimeter-wave access links to the user terminals. The small cells themselves will also need backhauling to the core network. This in

\footnotetext{
* Correspondence: richard.weiler@hhi.fraunhofer.de

${ }^{1}$ Fraunhofer Heinrich-Hertz-Institute, Berlin, Germany

Full list of author information is available at the end of the article
}

turn might also be deployed using the millimeter-wave bands for interference and bandwidth considerations. For the successful design and development of such systems, a comprehensive channel model that covers the relevant propagation effects is an essential basis.

The millimeter-wave band has already been used for fixed outdoor applications with success in the recent years [4]. The main concerns for this kind of applications are the impact of weather conditions (rain, snow, fog) and the availability of an unblocked lineof-sight (LOS) on the link quality. As the links are mostly static, simple path loss models are sufficient for these applications.

A large number of channel models have been proposed and used for sub- $6 \mathrm{GHz}$ wireless communication and different kinds of applications and use cases. A wellknown model for mobile radio networks is, e.g., the WINNER II channel model [5]. It relies on a geometrybased stochastic approach and was designed for frequencies from 2 to $6 \mathrm{GHz}$ with up to $100 \mathrm{MHz}$ bandwidth. Its parameters are determined stochastically, based on statistical distributions extracted from channel measurement data. The model was developed for a wide range of propagation scenarios ranging from indoor office, urban micro-cell to urban and rural macro-cell. Different scenarios are modeled by the same approach but with different parameters. When going to higher carrier frequencies in the millimeter-wave band and wider bandwidths, the WINNER II and similar geometry-based stochastic models might not be valid any more.

\section{Springer}


Recently, the widely used IEEE 802.11 standard for wireless local area communication has been extended to the $60-\mathrm{GHz}$ band with the 802.11ad protocol. This standard targets indoor communication. A new channel model was developed for this standard, based on the observation that the wireless channel can be well described with a set of distinct geometry-based propagation paths [6]. Spatial resolved measurements were performed in static indoor environments, showing that ray-tracing-like propagation paths with up to two reflections dominate the received power [7].

Here, we present the quasi-deterministic (Q-D) channel model and the link level-focused Canal Enregistré de Propagation Déterministe (CEPD) model, which have been developed and used within the Millimeter-Wave Evolution for Backhaul and Access (MiWEBA) project. The Q-D model combines a geometry-based approach for a limited number of multipath components and a stochastic approach. In this paper, we show how this channel modeling approach may be applied for specific access use cases (outdoor open square and large indoor area) and further how it can be modified for other environments. This modeling approach was chosen in order to accurately support spatial consistency that would not be possible with a statistical model. New experimental data for an open square scenario measurement are used to improve the modeling methodology described previously in $[8,9]$. An extension is given in a form of the CEPD model that can be used to abstract the generic Q$\mathrm{D}$ model to system level requirements, such as limited bandwidth and impact of antennas.

The development of the channel model was driven by a set of reference use cases and scenarios, defined in the MiWEBA project, whose focus is the investigation of new 5G architectures with millimeter-wave technology $[10,11]$. These use cases are similar to other 5G-related investigations, with a focus on millimeter-wave frequencies and their limitations. Apart from indoor and backhaul scenarios, emphasis is on outdoor mobile access links of small cell base stations with a typical cell radius of several hundred meters. The work presented in this paper focuses on this kind of links.

Section 2 details the performed outdoor access channel measurements to give an understanding of environment and the observed radio propagation effect. Section 3 then introduces the Q-D modeling approach. The measurement-based parameterization of the model is given in Section 4. Section 5 explains the link layer focus channel model and links it to the Q-D model.

\section{Open square scenario experimental measurements}

A measurement campaign was performed on "Leipziger Platz" in downtown Berlin, Germany [11]. This is an open square of octagonal shape with modern glass and stone buildings to the sides. A street with three lanes per direction is cutting through the square with a diameter of $150 \mathrm{~m}$. The area itself is covered with grass and some trees and lined with sidewalks, as can be seen in Figs. 1 and 2. The transmitter was placed at a height of $3.5 \mathrm{~m}$ at four different positions on the sidewalk and on the grass. This height was assumed as a starting point for small cell deployments being placed on existing street furniture. The receiver was moved along the sidewalk with an antenna height of $1.5 \mathrm{~m}$. The receiver tracks and their associated transmitter positions are colored alike.

The center frequency of the measurement campaign was $60 \mathrm{GHz}$ with a sounding bandwidth of $250 \mathrm{MHz}$. The antennas used on both sides are commercially available vertical polarized omni-directional antennas with a gain of $2 \mathrm{dBi}$. Their radiation pattern is flat in the entire azimuth as well as for elevation angles from $-30^{\circ}$ to $30^{\circ}$. This allows acquiring omni-directional power delay profiles without any mechanical steering. The influence on the antenna pattern in elevation is expected to be minimal, as there are no major sources of reflection outside the $60^{\circ}$ half-power opening angle.

The receiver cart is either static or moving at a constant speed of $0.5 \mathrm{~m} / \mathrm{s}$, each with a temporal snapshot separation of $800 \mu \mathrm{s}$. Due to properties of the measurement system, the maximum number of snapshots per acquisition is ca. 60.000 . Tracks requiring longer acquisition are measured in multiple adjacent runs.

Due to the measurement bandwidth and the geometry of LOS and ground-reflected propagation, fading can occur on the measured path power [12]. Averaging allows reducing this effect and simplifies measurements, but for the data analysis in the present paper, no averaging is applied, as the focus lies on the multipath properties of the channel detailed investigation.

A typical channel impulse response vs. time plot on the sidewalk at the middle road ( $\mathrm{Tx} 1$, receiver at $\mathrm{P} 1$ ) is given in Fig. 3. The LOS path is clearly visible and stable at a delay of $84 \mathrm{~ns}$, which equals exactly the transmitter to receiver distance of $25 \mathrm{~m}$ in this measurement. The ground reflection cannot be resolved due to the measurement bandwidth. Other stable propagation paths at higher delays are clearly visible. For example, in Fig. 3 there are strong components at 276, 308, and 324-ns delays, being path lengths of ca. 83, 92, and $97 \mathrm{~m}$ respectively. It should be noted that power fluctuation of those stable components in time was less than 1-2 dB. Figure 4 shows an average power delay profile of an entire 50-s measurement run at the same location. Note that this figure shows the full resolvable delay axis up to $1.024 \mu \mathrm{s}$, opposed to the previous figure that was restricted to $400 \mathrm{~ns}$. 


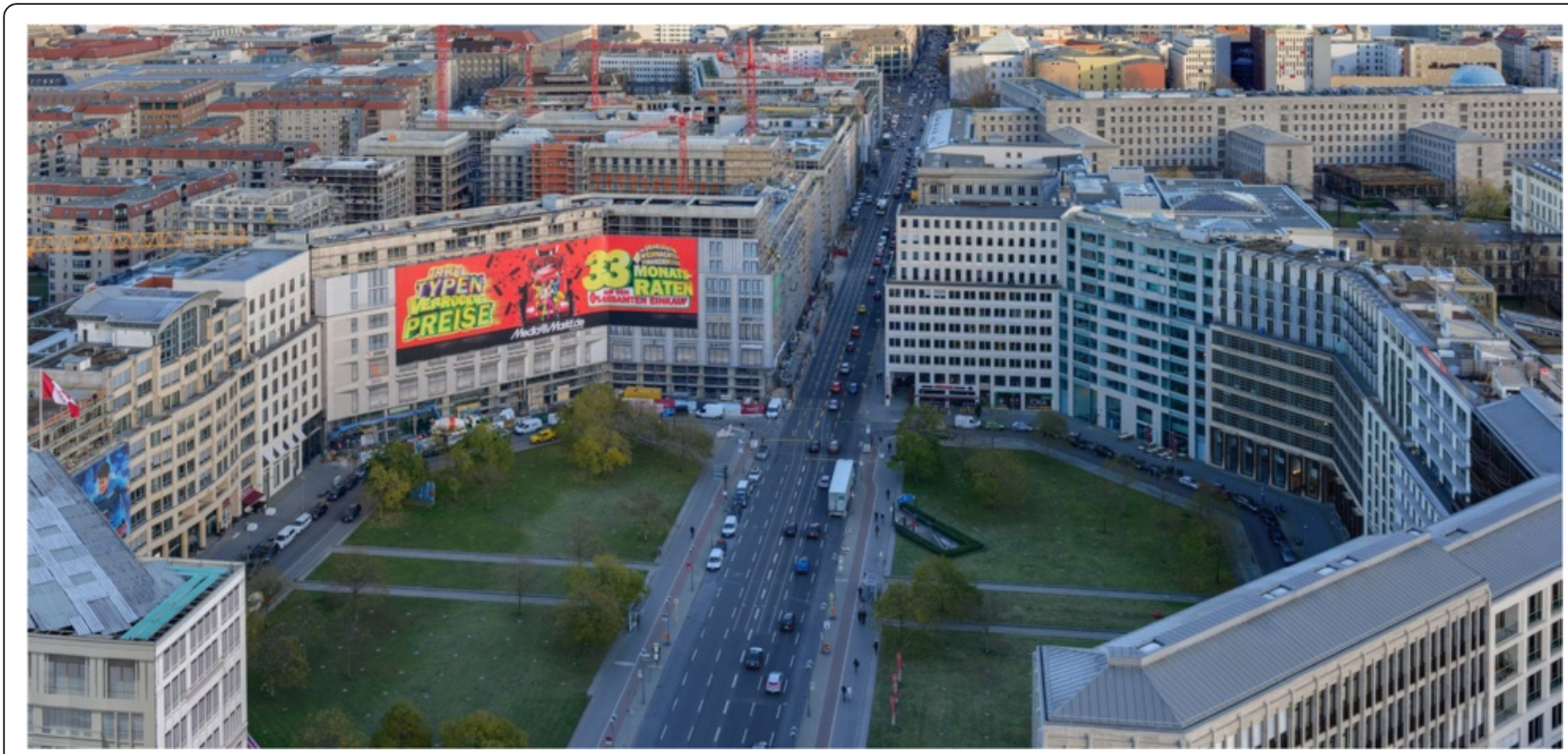

Fig. 1 Leipziger Platz measurement site view (Photo: Arild Vågen, License: CC BY-SA 3.0)

Channel impulse responses for moving receivers are shown in Figs. 5 and 6. In the first case, the transmitter is placed at the position Tx2 and the receiver is moving along track Rx2, starting at the western end. In the second figure, the transmitter is placed on the position Tx4 and the receiver is moving along the dashed part of the Rx4 track, starting at the northern end. Multiple adjacent measurement runs (three and two, respectively) were concatenated for the plots, causing some minor discontinuities. The LOS delay varies in both cases as

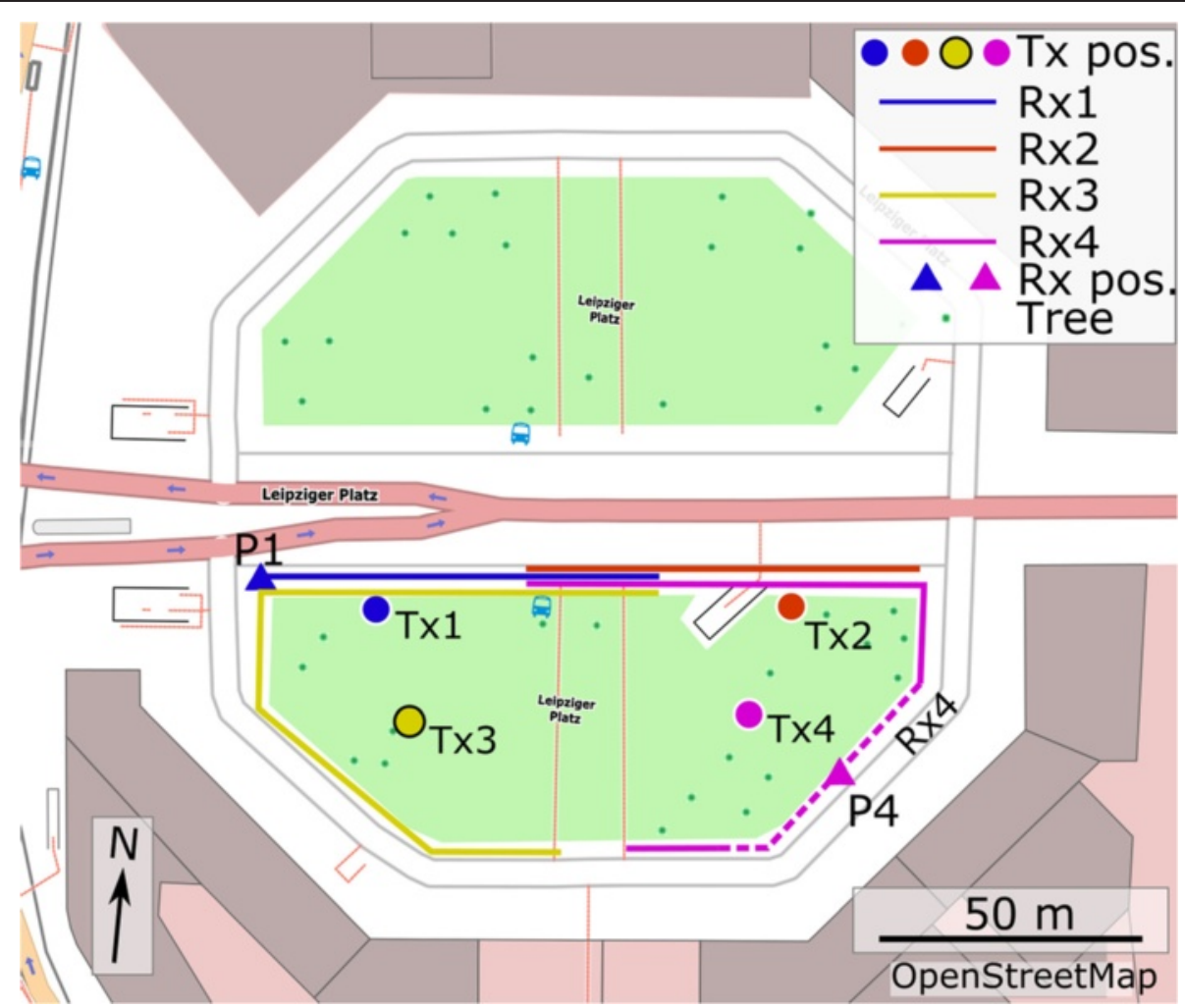

Fig. 2 Map of open square measurements 


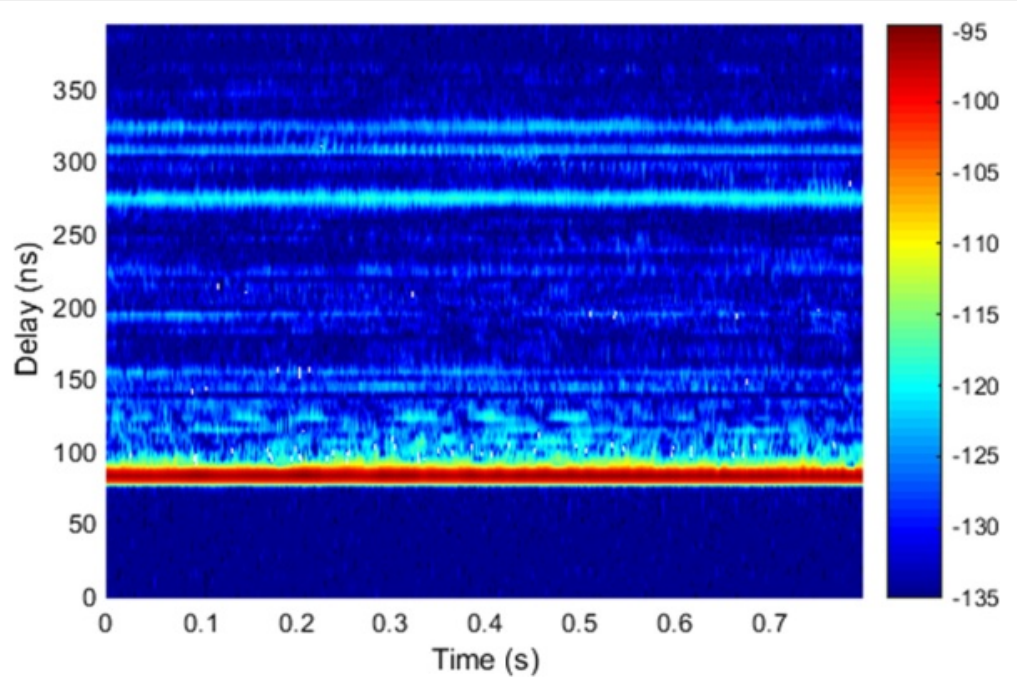

Fig. 3 Channel impulse response with a static receiver, Tx1

the receiver is moving closer and farther from the transmitter location, matching well with the geometrical $\mathrm{Tx}$ $\mathrm{Rx}$ distance. In the Tx4 case, a strong multipath component is visible at an excess delay (over LOS) of ca. $25 \mathrm{~ns}$. This path could be identified as a reflection from the closest building. On this reflected component, a strong fading effect is visible, but as described earlier, this can be caused by the reflected ray and its ground reflection, which cannot be resolved with the measurement bandwidth. In the Tx2 measurement, the next building wall is much more distant and therefore much weaker. On the other hand, some multipath components become visible at ca. $80 \mathrm{~s}$ when the receiver passes the subway exit with its metallic construction. As with the static measurement, a number of stable multipath components (clearly visible as lines at the time-delay diagram) are caused by surrounding buildings. The "color noise" also present on the diagram is caused by reflections from various objects such as trees and street furniture. The delay of these paths depends on the precise geometrical setup of the environment. Analyzing the channel impulse response at greater delays than shown here reveals that the strength of these components is much weaker and quickly vanishes in the dynamic range of the measurement. The maximum observed relevant delay relative to LOS path is about $150-200 \mathrm{~ns}$.

Another static average power delay profile is given in Fig. 7. The receiver position corresponds to a time of $45 \mathrm{~s}$ in Fig. 6. In this position, the multipath component with an excess delay of $50 \mathrm{~ns}$ is about $20 \mathrm{~dB}$ below the

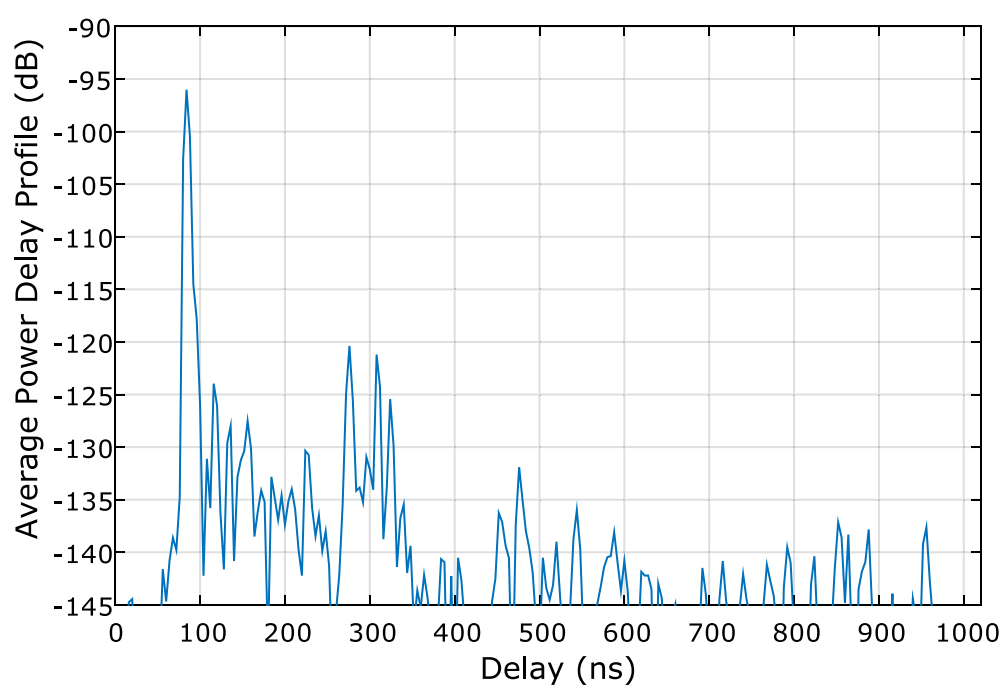

Fig. 4 Average power delay profile of static receiver pos. P1 with Tx1 


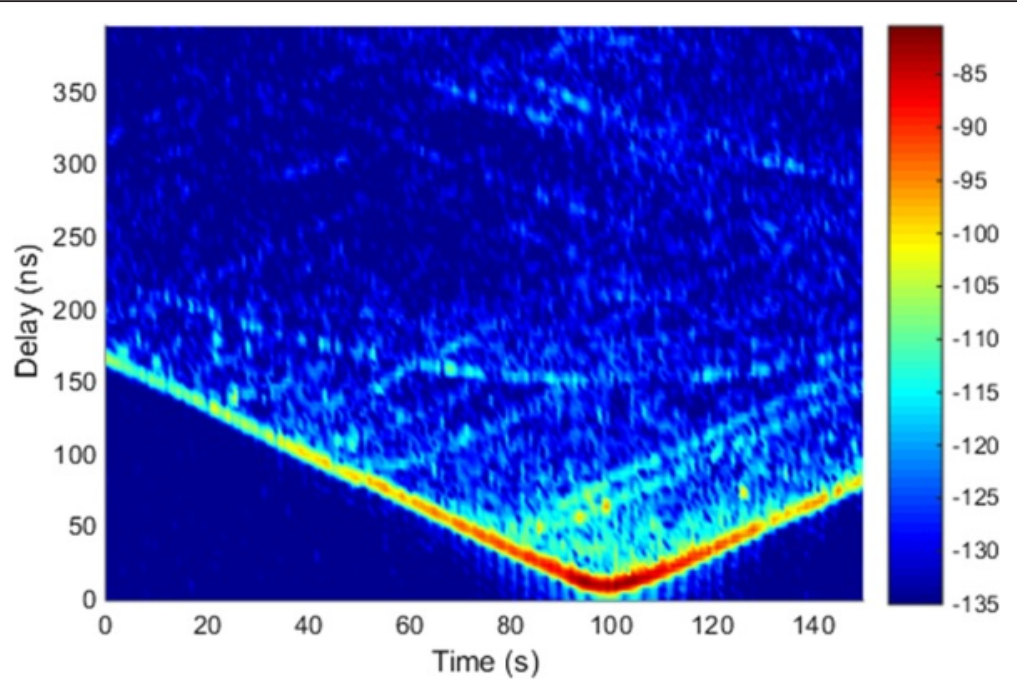

Fig. 5 Channel impulse response with a moving receiver, Tx2

LOS, but another component with an excess delay of ca. $110 \mathrm{~ns}$ is visible, which is less than $10 \mathrm{~dB}$ below the LOS power. The measurement results for the Tx position Tx3 and the Rx position Rx3 are very similar to the Tx4 and Rx4 results, respectively.

The analysis of other channel parameters from these and similar urban access measurements, such as path loss and delay spread, was presented in earlier work [13, 14].

\section{Quasi-deterministic channel model}

The experimental measurement results in Section 2, represented in the form of time-delay diagrams (Figs. 3, 4, 5,6 , and 7), illustrate the fact that the channel for static and moving $\mathrm{Tx}-\mathrm{Rx}$ positions is not completely random, but has some Q-D components, that are represented in the time-delay diagrams by steady, clearly visible lines and traces. Moreover, the strongest traces can be identified as LOS path and reflections from the nearest buildings. The same diagram, plotted by using the ray-tracing reconstruction of the environment, will be very similar to the one seen in the experiments, with difference only in noise-like background and weak short traces that can be caused by small objects. These observations lead to the conclusion that realistic millimeter-wave channel models can consist of deterministic components, defined by the scenario and random components, representing unpredictable factors or objects that are random or insignificant.

Such an approach, called Q-D, was offered for modeling access and backhaul millimeter-wave channels at $60 \mathrm{GHz}[9,15]$. The approach builds on the

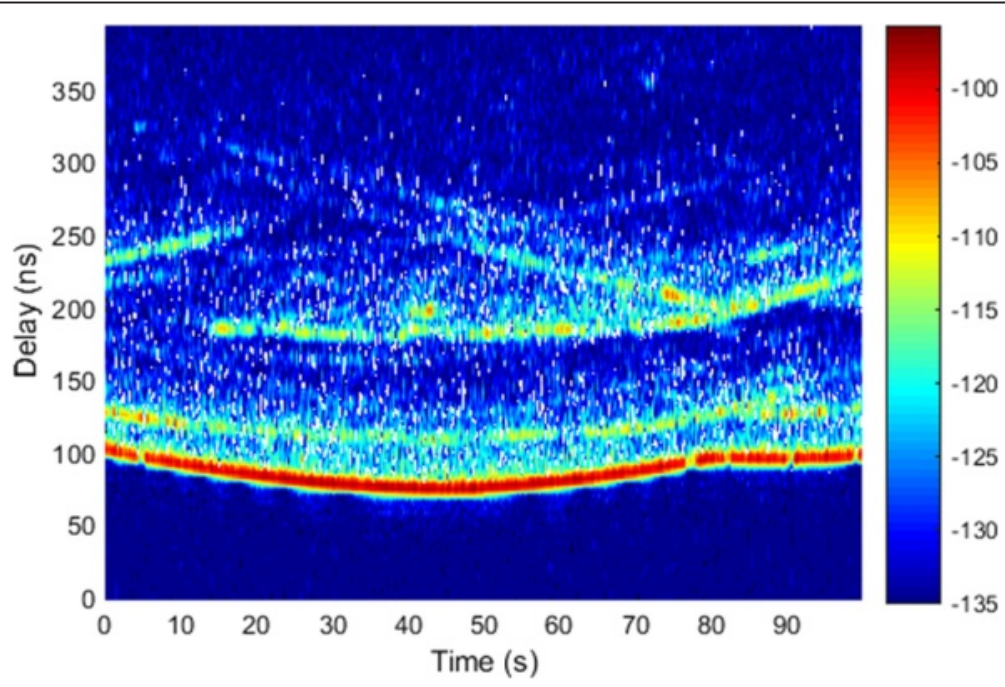

Fig. 6 Channel impulse response with a moving receiver, Tx4 


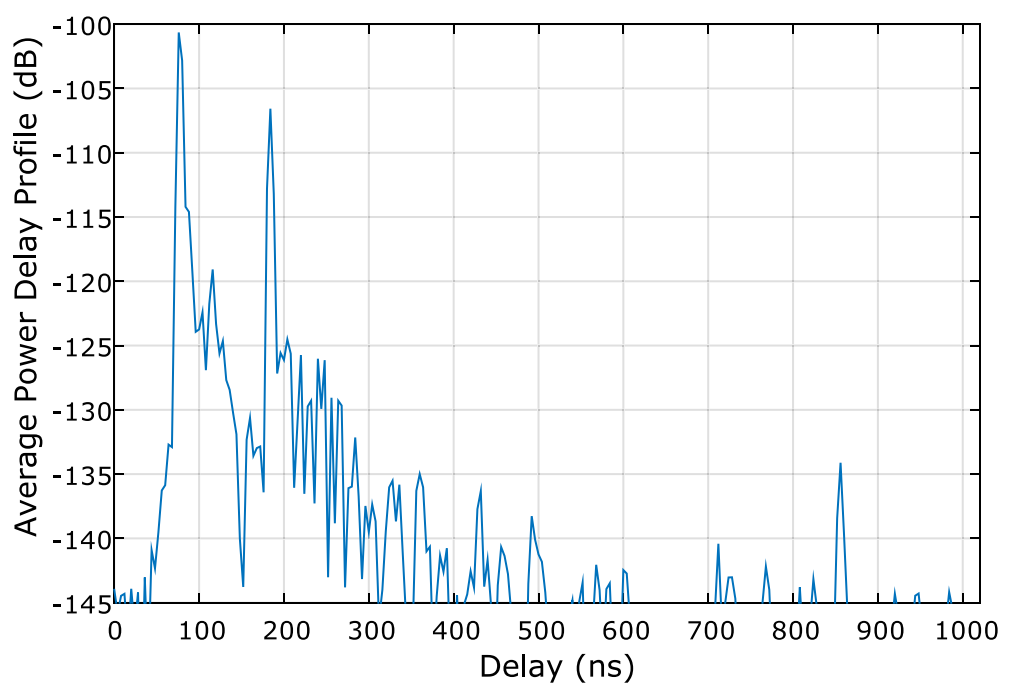

Fig. 7 Average power delay profile of static receiver pos. P4 with Tx4

representation of the millimeter-wave channel impulse response comprised of a few Q-D strong rays (D-rays), a number of relatively weak random rays (R-rays, originating from the static surfaces reflections), and flashing rays (F-rays, originating from moving cars, buses, and other dynamic objects reflections).

The key benefit of this approach compared to pure statistical models is its inherent support for spatial consistency. The deterministic part of the channel impulse response accurately takes the position of the transmitter and receiver into account. Simulating a moving user, for example, the band-limited channel impulse response can accurately reproduce fading effects, observed in real measurements [16]. This is not possible with a purely statistical model.

The first type of rays makes the major contribution into the signal power, is present all the time, and usually can be clearly identified as a reflection from scenarioimportant macro objects. It is logical to include them into the channel model as deterministic (D-rays), explicitly calculated values. The element of randomness, important for the statistical channel modeling, may be introduced on the intra-cluster level, by adding a random exponentially decaying cluster to the main D-ray.

The second type of rays (R-rays) is the reflections from the random objects or the objects that is not mandatory in the scenario environment. Such type of rays may be included in the model in a classical statistical way, as rays with parameters (power and delays) selected randomly in accordance with the predefined distributions.

The third type of rays (F-rays) may be introduced to the model in the same way as R-rays but with some additional statistic for appearing chance and duration.
All the types of rays are then combined in the singleclustered channel impulse response, shown in Fig. 8. Here, a cluster refers to multipath components with similar delay, angle of departure (AoD), and angle of arrival (AoA) parameters. All of these parameters must be similar in order for the multipath components to form a cluster, and paths belonging to a cluster should have the same physical propagation mechanisms [5].

For each of the channel propagation scenarios, the strongest propagation paths are determined and associated to rays which produce the substantial part of the received useful signal power. Then, the signal propagation over these paths is calculated based on the geometry of the deployment and the locations of the transmitter and receiver, calculating the ray parameters, such as AoA and AoD, power, and polarization characteristics. The signal power conveyed over each of the rays is calculated in accordance to theoretical formulas taking into account free-space losses, reflections, antenna polarization, and receiver mobility effects like Doppler shift. Some of the parameters in these calculations may be considered as random values like reflection coefficients or as random processes like receiver motion. The number of D-rays, which are taken into account, is scenario dependent and is chosen to be in line with the channel measurement results. Additionally to the D-rays, a lot of other reflected waves are received from different directions, coming, for example, from cars, trees, lamp posts, benches, and houses (for outdoor scenarios) or from room furniture and other objects (for indoor scenarios). These rays are modeled as R-rays. These rays are defined as random clusters with specified statistical parameters extracted from available experimental data or ray-tracing modeling. 


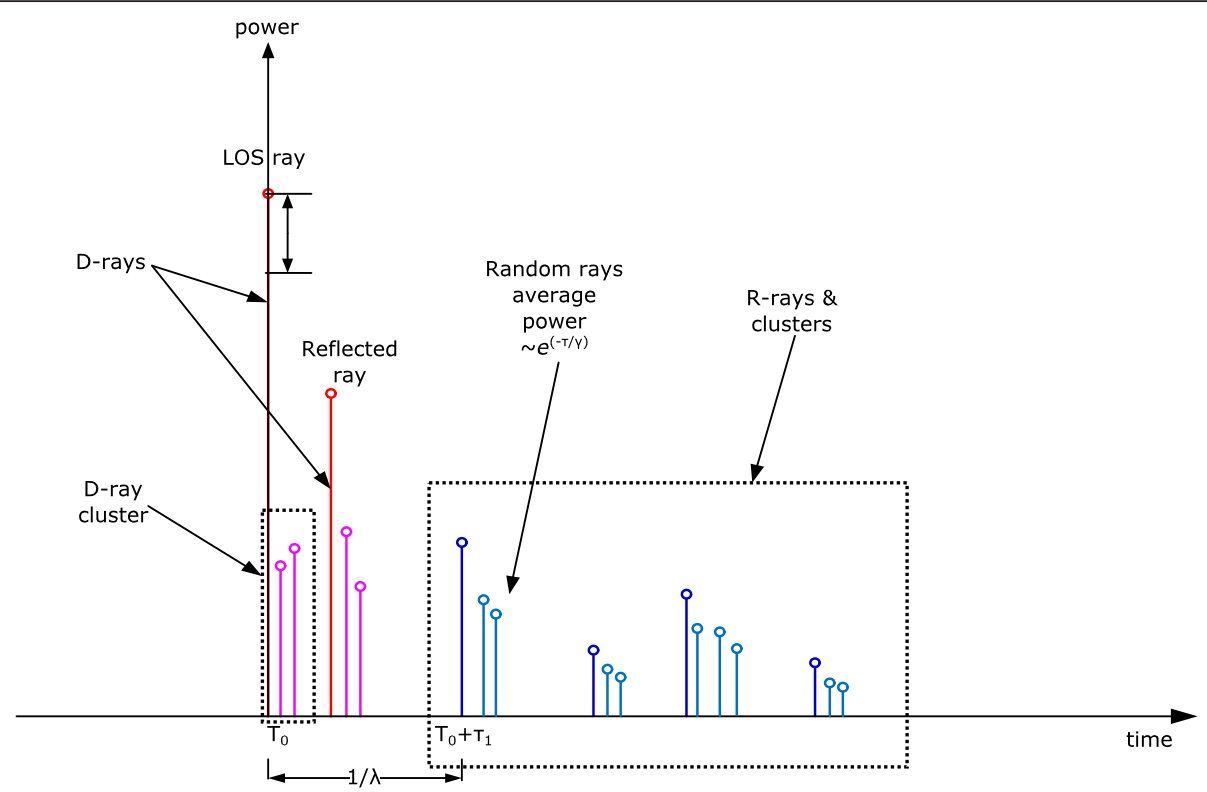

Fig. 8 Q-D channel model channel impulse response structure

For a given environmental scenario, the process of the definition of D-rays and R-rays and their parameters is based both on experimental measurements and raytracing reconstruction of the environment. The results presented in Section 2 on experimental measurement may serve as a great example of the process. The experimental measurement processing includes peak detection algorithm [15] with further accumulation of the peak statistics over time, identifying the percentage of the selected ray activity during observation period. The rays with activity percentage above $80-90 \%$ are the D-rays: strong and always present, if not blocked. The blockage percentage for D-rays may be estimated around 2-4\%. The rays with activity percentage about $40-70 \%$ are the R-rays: the reflections from far-away static objects, weaker, and more susceptible to blockage due to longer travel distance. And finally, the rays with activity percentage below $30 \%$ are the F-rays: the flashing reflection from random moving objects. Such rays are not "blocked"; they actually "appear" only for a short time.

Scenarios with obstructed line-of-sight (OLOS) between the transmitter and receiver antennas, caused by moving or fixed objects (e.g., cars, pedestrians, trees), can be integrated in the model with a stochastic process. Scenarios where the LOS is completely blocked by large objects (e.g., buildings) are often referred to as non-lineof-sight (NLOS). These might also be taken into account by the model, by blocking the LOS and ground reflection D-ray permanently and by carefully defining the other D-rays. Measurements have to be performed to derive meaningful parameters for this case. The measurement campaign described in Section 2 focused on
LOS and OLOS scenarios and does not provide information on this. Other measurements performed by the authors however show that a millimeter-wave signal can be received in an urban street canyon NLOS scenario, but received signal strength quickly drops with the distance [14].

A clustered structure is added as a final stage for both for Q- and R-rays, introducing the set of exponentially decaying rays after the main ray of the cluster, with the pre-defined K-factor and time of arrival distribution. The resulting channel impulse response can be represented as the sum of clusters corresponding to D-rays (direct ray, ground reflection, etc.), clusters corresponding to R-rays, and clusters corresponding to F-rays: see expression (1). For taking into account the polarization effects, the square channel matrix $\mathbf{H}^{i}$ is introduced instead of scalar, modeling both polarizations and their dependence.

$$
\begin{aligned}
\boldsymbol{h}\left(t, \varphi_{t x}, \theta_{t x}, \varphi_{r x}, \theta_{r x}\right)= & \sum_{i} \mathbf{H}^{i} C^{i}\left[t-T^{i}, \varphi_{t x}-\Phi_{t x}^{i},\right. \\
& \left.\theta_{t x}-\Theta_{t x}^{i}, \varphi_{r x}-\Phi_{r x}^{i}, \theta_{r x}-\Theta_{r x}^{i}\right] \\
C^{i}\left(t, \varphi_{t x}, \theta_{t x}, \varphi_{r x}, \theta_{r x}\right)= & \sum_{k} \alpha^{i, k} \delta\left(t-\tau^{i, k}\right) \delta\left(\varphi_{t x}-\varphi_{t x}^{i, k}\right) \\
& \times \delta\left(\theta_{t x}-\theta_{t x}^{i, k}\right) \delta\left(\varphi_{r x}-\varphi_{r x}^{i, k}\right) \\
& \times \delta\left(\theta_{r x}-\theta_{r x}^{i, k}\right)
\end{aligned}
$$

where $\boldsymbol{h}$ is a generated total channel impulse response function and $t$ is current time; $\varphi_{t x}, \theta_{t x}, \varphi_{r x}, \theta_{r x}$ are azimuth and elevation angles at the transmitter and receiver, respectively; $\mathbf{H}^{i}$ and $C^{i}$ are the gain matrix and the channel impulse response function for the $i$-th 
cluster, respectively, $\delta()$ is the Dirac delta function; $T^{i}$, $\Phi_{t x}{ }^{i}, \Theta_{t x}{ }^{i}, \Phi_{r x}{ }^{i}, \Theta_{r x}{ }^{i}$ are time-angular coordinates of the $i$-th cluster; $\alpha^{i, k}$ is the amplitude of the $k$-th ray of the $i$-th cluster; and $\tau^{i, k}, \varphi_{t x}^{i, k}, \theta_{t x}^{i, k}, \varphi_{r x}{ }^{i, k}, \theta_{r x}^{i, k}$ are relative timeangular coordinates of the $k$-th ray of the $i$-th cluster.

Note that each ray has its own delay $\tau$, angular characteristics, such as $\operatorname{AoD}\left(\varphi_{t x}, \theta_{t x}\right)$, AoA $\left(\varphi_{r x}, \theta_{r x}\right)$, and, finally, the channel matrix $\mathbf{H}$ that characterizes the polarization, power, and phases of the two polarization components. In this case, the transmission equation for a single-ray channel may be written as:

$$
y=G_{t x}\left(\varphi_{t x}, \theta_{t x}\right) G_{r x}\left(\varphi_{r x}, \theta_{r x}\right) \boldsymbol{e}_{r x}^{H} \mathbf{H} \boldsymbol{e}_{t x} x
$$

where $x$ and $y$ are the transmitted and received signals, $\boldsymbol{e}_{t x}$ and $\boldsymbol{e}_{r x}$ are the polarization (Jones) vectors for the Tx and $\mathrm{Rx}$ antennas, respectively, and $G_{t x}(\varphi, \theta)$ and $G_{r x}(\varphi, \theta)$ are antenna gains at given angular coordinates. Generally, the $G_{t x}$ and $G_{t x}$ are different for different polarizations and should be represented as vectors, just like $\boldsymbol{e}_{t x}$ and $\boldsymbol{e}_{r x}$.

\section{Q-D channel model development}

The analysis of experimental measurements described in Section 2 and the results available from previous experimental campaigns $[9,17]$ have shown that the Q-D channel model with multiple $\mathrm{D}$-rays may provide appropriate description for the different environments. Based on the Q-D methodology, the millimeter-wave channel model for an open area (open square, university campus, etc.) scenario was developed. Figure 9 illustrates the environment setup with two D-rays (direct ray and ground-reflected ray) with a number of R-rays reflected from random objects in the area. But, as shown in Section 2, the open square scenario may have a few additional strong rays, stemming from different objects, such as metalized bus stop windows and surrounding buildings. To have a more accurate scenario description, these strongest reflections can be taken into account explicitly as additional D-rays, but weaker paths should be included in a statistical manner, as R-rays.

\subsection{D-ray modeling}

The Q-D rays are explicitly calculated in accordance with scenario parameters, geometry, and propagation conditions. The propagation loss is calculated by the Friis equation, taking additional losses from the oxygen absorption into account (Table 1, second row). An important part of the proposed Q-D approach to the channel modeling is the calculation of the reflected ray parameters. The calculations are based on the Fresnel equations, with additionally taken into account losses due to surface roughness (Table 2, second row)

The feasibility of the proposed approach to the prediction of the signal power is proven in [18] for outdoor micro-cell environments and in [19] and [20] for intervehicle communication modeling. In general, problems of the signal power prediction are considered in [21].

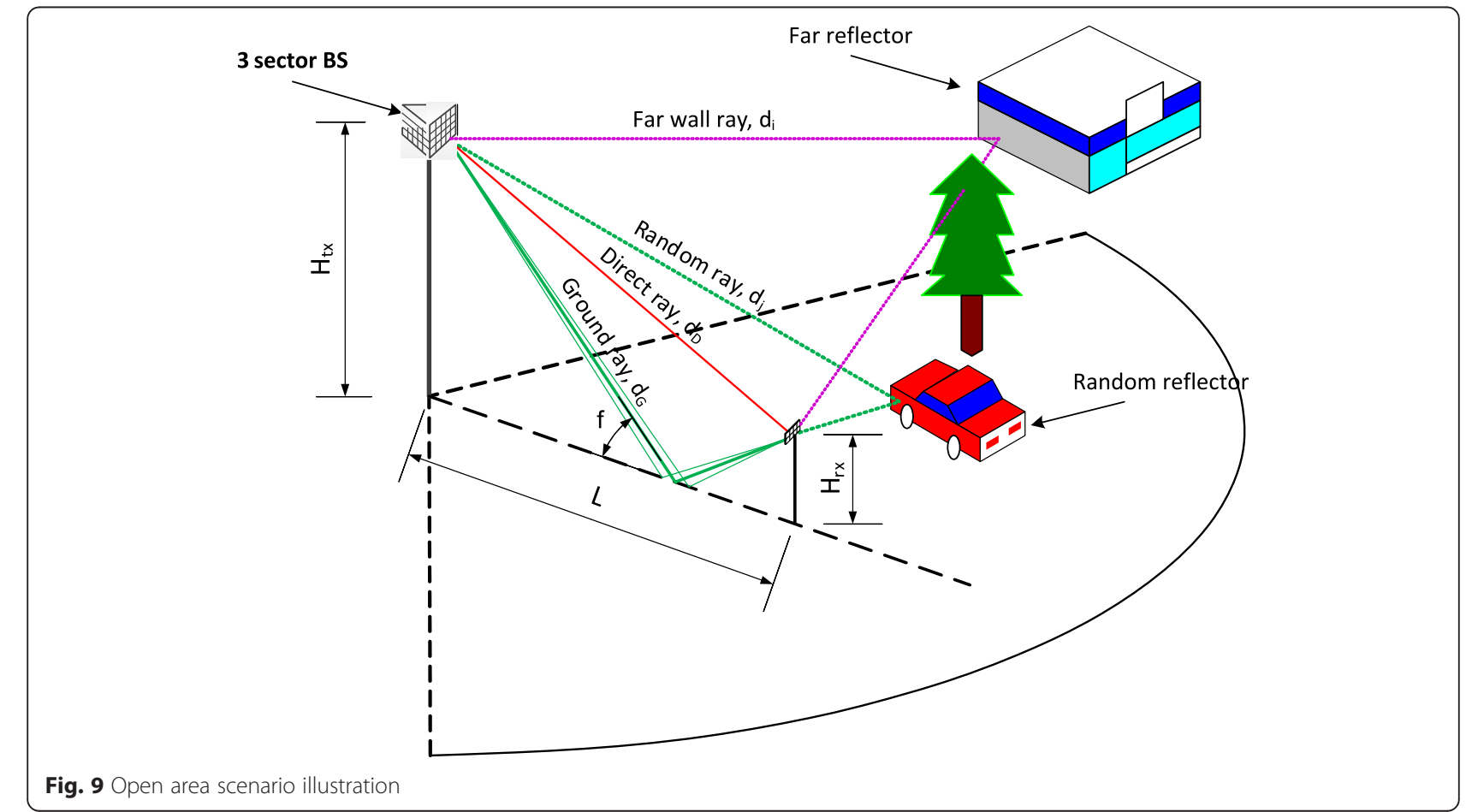


Table 1 Direct ray parameters

\begin{tabular}{ll}
\hline Parameter & Value \\
\hline Delay & Direct ray delay is calculated from the model \\
& geometry: $T_{D}=d_{D} / C$ \\
& $d_{D}=\sqrt{L^{2}+\left(H_{t x}-H_{r x}\right)^{2}}$ \\
& Direct ray power calculated as free-space path \\
Power & loss with oxygen absorption: \\
& $P_{D}=20 \log _{10}\left(\frac{\lambda}{4 \pi d_{D}}\right)-A_{0} d_{D}$, in dB \\
Channel matrix & $\mathbf{H}=\left[\begin{array}{cc}10^{P_{D} / 20} & 0 \\
0 & 10^{P_{D} / 20}\end{array}\right] e^{\frac{2 \pi d_{D}}{\lambda}}$ \\
AoD & $0^{\circ}$ azimuth and elevation \\
AoA & $0^{\circ}$ azimuth and elevation \\
\hline
\end{tabular}

$A o D$ angle of departure, $A \circ A$ angle of arrival

The D-rays are strictly scenario dependent, but in all considered scenarios, two basic D-rays are present: the direct LOS ray and the ground-reflected ray. The calculation of those two basic rays' parameters will be the same for all scenarios.

\subsubsection{Direct ray}

The direct LOS ray is a ray between $\mathrm{Tx}$ and $\mathrm{Rx}$.

\subsubsection{Ground ray}

The ground-reflected ray presents in all considered scenarios. Its parameters are calculated based on the Friis free-space path loss equation and the Fresnel equation to take into account reflection and rough surface

Table 2 Ground-reflected ray parameters

\begin{tabular}{|c|c|}
\hline Parameter & Value \\
\hline Delay & $\begin{array}{l}\text { Ground-reflected ray delay is calculated from the } \\
\text { model geometry: } \\
\tau_{G}=d_{G} / c \\
d_{G}=\sqrt{L^{2}+\left(H_{t x}+H_{r x}\right)^{2}}\end{array}$ \\
\hline Power & $\begin{array}{l}\text { Ground-reflected power calculated as free-space } \\
\text { path loss with oxygen absorption, with additional } \\
\text { reflection loss calculated on the base of the Fresnel } \\
\text { equations. Reflection loss } R \text { is different for vertical } \\
\text { and horizontal polarizations } \\
P_{\perp}=20 \log _{10}\left(\frac{\lambda}{4 \pi d_{G}}\right)-A_{0} d_{G}+R_{\perp}+F ; \\
P_{\|}=20 \log _{10}\left(\frac{\lambda}{4 \pi d_{G}}\right)-A_{0} d_{G}+R_{\|}+F \\
F=80 / \ln 10\left(\pi \sigma_{h} \sin \phi / \lambda\right)^{2} \\
R_{\perp}=20 \log _{10}\left(\frac{\sin \phi-\sqrt{B_{\perp}}}{\sin \phi+\sqrt{B_{\perp}}}\right) ; R_{\|}=20 \log _{10}\left(\frac{\sin \phi-\sqrt{B_{\|}}}{\sin \phi+\sqrt{B_{\|}}}\right) \\
B_{\|}=\varepsilon_{r}-\cos ^{2} \phi \text { for horizontal polarization. } \\
B_{\perp}=\left(\varepsilon_{r}-\cos ^{2} \phi\right) / \varepsilon_{r}^{2} \text { for vertical polarization, } \\
\text { where } \tan \phi=\frac{H_{t x}+H_{r x}}{L} \text { and } \sigma_{h} \text { is a surface roughness. }\end{array}$ \\
\hline $\begin{array}{l}\text { Channel } \\
\text { matrix }\end{array}$ & $\mathbf{H}=\left[\begin{array}{cc}10^{P_{\perp} / 20} & \xi \\
\xi & 10^{P_{\|} / 20}\end{array}\right] e^{\frac{j 2 \pi d_{G}}{\lambda}}$ \\
\hline AoD & Azimuth: $0^{\circ}$, elevation: $\theta_{A O D}=\tan ^{-1}\left(\frac{L}{H_{I_{x}}-H_{I_{x}}}\right)-\tan ^{-1}\left(\frac{L}{H_{I^{x}}+H_{I_{x}}}\right)$ \\
\hline AoA & Azimuth: $0^{\circ}$, elevation: $\theta_{A O A}=\tan ^{-1}\left(\frac{H_{x x}+H_{x x}}{L}\right)-\tan ^{-1}\left(\frac{H_{x x}-H_{x x}}{L}\right)$ \\
\hline
\end{tabular}

$A \circ D$ angle of departure, $A \circ A$ angle of arrival scattering factor $F$. Note that the horizontally and vertically polarized components of the transmitted signal will be differently reflected and, thus, the channel matrix should have different diagonal elements.

\subsubsection{Additional rays}

For the open area scenario, with no significant reflection objects other than ground, only two D-rays are considered. However, in more rich scenarios, like the one considered here as the large square, or, for example, street canyon scenario, reflection from one or more walls should be taken into account. The principle of calculation of these additional D-rays is the same; detailed description may be found in [15]. The closest wall can be calculated using the geometry and positions of the transmitter and receiver. The calculation of the path properties is equal to the ground ray reflection in the previous section with adapted material parameters.

\subsection{R-ray modeling}

For taking into account a number of rays that cannot be easily described deterministically (reflections from objects that are not fully specified in the scenario, objects with random or unknown placement, objects with complex geometry, higher-order reflections, etc.), the statistical approach is used in the Q-D channel modeling methodology. The clusters arrive at moments $\tau_{k}$ according to the Poisson process and have inter-arrival times that are exponentially distributed. The cluster amplitudes $A\left(\tau_{k}\right)$ are independent Rayleigh random variables, and the corresponding phase angles $\theta_{k}$ are independent uniform random variables over $[0,2 \pi]$.

The R-ray components of the channel impulse response are given by:

$$
h_{\text {cluster }}(t)=\sum_{k=1}^{N_{\text {cluster }}} A\left(\tau_{k}\right) e^{j \theta_{k}} \delta\left(t-\tau_{k}\right),
$$

where $\tau_{k}$ is the arrival time of the $k$-th cluster measured from the arrival time of the LOS ray and $A\left(\tau_{k}\right), P\left(\tau_{k}\right)$, and $\theta_{k}$ are the amplitude, power, and phase of the $k$-th cluster, respectively. The R-rays are random, with Rayleighdistributed amplitudes and random phases, with exponentially decaying power delay profile. The total power is determined by the K-factor with respect to the direct LOS path.

$$
\begin{aligned}
& P\left(\bar{\tau}_{k}\right)=P_{0} e^{-\tau_{k} / \gamma} \\
& \frac{P_{L O S}}{\sum P\left(\tau_{k}\right)}=K
\end{aligned}
$$

Table 3 summarizes the R-ray parameters for the open area/large square models. The power delay profile parameters are derived based on the available experimental data and corresponding ray-tracing simulations. The 
Table 3 Open square model R-ray parameters

\begin{tabular}{ll}
\hline Parameter & Value \\
\hline Number of rays, $N$ & 3 \\
Poisson arrival rate, $\lambda$ & $0.05 \mathrm{~ns}^{-1}$ \\
Power decay constant, $\gamma$ & $15 \mathrm{~ns}$ \\
$K$-factor & $6 \mathrm{~dB}$ \\
AoA & Elevation: $\cup\left[-20: 20^{\circ}\right]$ \\
& Azimuth: $\cup\left[-180: 180^{\circ}\right]$ \\
AoD & Elevation: $U\left[-20: 20^{\circ}\right]$ \\
& Azimuth: $U\left[-180: 180^{\circ}\right]$ \\
\hline
\end{tabular}

$A o D$ angle of departure, $A \circ A$ angle of arrival

AoA and AoD ranges illustrate the fact that random reflectors can be found anywhere around the receiver but are limited in height. Uniform distributions are selected for simplicity and can be further enhanced on the base of more extensive measurements.

In the 802.11ad channel model [6], the set of approximations was proposed for diagonal and off-diagonal elements of the channel matrix $\mathbf{H}$ for the first- and secondorder reflections in typical indoor environments (conference room, cubicle, and living room) as a combination of log-normal and uniform distributions on the base of experimental studies [22]. In the Q-D model, the ray amplitude is approximated by the Rayleigh distribution (which is close to log-normal) so that the simple fixed polarization matrix $\mathbf{H}_{\mathrm{p}}$ may be used for introducing polarization properties to the R-rays (matrix $\mathbf{H}$ is obtained by multiplication of the scalar amplitudes $A$ to the polarization matrix $\mathbf{H}_{\mathrm{p}}$ ). The polarization matrix $\mathbf{H}_{\mathrm{p}}$ for R-rays is defined by:

$$
\mathbf{H}_{p}=\left[\begin{array}{cc}
1 & \pm 0.1 \\
0.1 & \pm 1
\end{array}\right]
$$

The values with sign \pm are assumed to have a random sign $(+1$ or -1 , for instance), with equal probability, independently from other values. For the cluster rays with the main R-ray, the polarization matrix is the same as the R-ray.

Flashing rays, or F-rays introduced in Section 3, are intended to describe the reflections from fast moving objects like vehicles and are short in duration. Its properties require additional investigations and analyses; thus, the F-rays are not included in the considered Q-D modeling approach application example.

\subsection{Intra-cluster structure modeling}

The surface roughness and presence of the various irregular objects on the considered reflecting surfaces and inside them (bricks, windows, borders, manholes, advertisement boards on the walls, etc.) lead to separation the specular reflection ray to a number of additional rays with close delays and angles: a cluster. The intra-cluster parameters of the channel model were extracted from the indoor models [6, 7], obtained from the measurement data [23]. The intracluster structure is introduced in the Q-D model in the same way as R-rays: as Poisson-distributed in time, exponentially decaying Rayleigh components, dependent on the main ray.

The identification of rays inside of the cluster in the angular domain requires very high angular resolution. The "virtual antenna array" technique where a low directional antenna element is used to perform measurements in multiple positions along the virtual antenna array to form an effective antenna aperture was in the MEDIAN project [24, 25]. These results were processed in [26], deriving the recommendation to model the intra-cluster angle spread for azimuth and elevation angles for both the transmitter and receiver as independent normally distributed random variables with zero mean and root mean square (RMS) equal to $5^{\circ}, N\left(0,5^{\circ}\right)$.

Note that it is reasonable to assume that different types of clusters may have distinctive intra-cluster structure. For example, properties of the clusters reflected from the road surface are different from the properties of the clusters reflected from brick walls because of the different material surface structures. Also, one may assume the properties of the first- and second-order reflected clusters to be different, with the second-order reflected clusters having larger spreads in temporal and angular domains. All these effects are understood to be reasonable. However, since the number of available experimental results was limited, a common intra-cluster model for all types of clusters was developed. Modifications with different intra-cluster models for different types of clusters may be a subject of the future channel model enhancements. The parameters are summarized in Table 4.

\section{Link level-focused channel model}

The link level-focused propagation channel model presented in this section is a multipath propagation

Table 4 Open square model intra-cluster parameters

\begin{tabular}{ll}
\hline Parameter & Value \\
\hline Intra-cluster ray K-factor & $6 \mathrm{~dB}$ for LOS ray, 4 dB for NLOS \\
Power decay time & $4.5 \mathrm{~ns}$ \\
Arrival rate & $0.31 \mathrm{~ns}^{-1}$ \\
Amplitude distribution & Rayleigh \\
Number of post-cursor rays & 4 \\
\hline
\end{tabular}

LOS line-of-sight, NLOS non-line-of-sight 
model dedicated to link level simulations. The Q-D model as well as experimental multipath channel impulse response files may be used as inputs of the model for link level assessments generating appropriate channel impulse responses (CIRs) fit with the simulated physical (PHY) layer and the considered propagation scenario. The link level-focused propagation channel model, also denoted CEPD model, is a multipath propagation model which conjunctly exploits multi-rate digital filter processing [27] and experimental multipath measurements to generate propagation CIRs $h(t, \tau)$ with scalable limited bandwidth and clocking rates. When simulating propagation, resampling is required in accordance with the simulated PHY layer. The model generates link level propagation CIRs using multi-rate filter processing to resample and filter the measured propagation channel, adapted to the PHY waveform of the system and simulated use cases [28]. Antenna alignment mismatch test cases allow quantized link level degradation assessments, when the antennas are not aligned. Analytical models are derived from an extension of the multi-slope model [29], describing antenna alignment mismatching effect on multipath channel, using dedicated measurements and CEPD realizations.

Multi-rate filter processing performs a $2 \times 1 \mathrm{D}$ conversion rate geared to relative delay $\tau$ and time $t$ in order to simulate the multipath propagation channel within the PHY bandwidth of the simulated system at the targeted system sampling rate and appropriate refreshment rate of the CIRs depending on environment topologies and time variations. The complex envelope of the time variant CIR of the propagation channel, $h(t, \tau)$, is described by two independent variables, typically the relative delay $\tau$ and the time $t$ as expressed below:

$$
\begin{aligned}
h(t, \tau) & =\sum_{k=1}^{N_{t}} a_{k}(t) \cdot \delta\left(\tau-\tau_{k}(t)\right) \rightarrow h(n, k) \\
& =\sum_{k=1}^{N_{t}} a_{k}(n) \cdot \delta(\tau-k / F s i g)
\end{aligned}
$$

$a_{k}(t)$ is the time variant amplitude of the relative delay $\tau_{k}(t)$. Time variant amplitudes $a_{k}(t)$ are assigned to echoes equally sampled depending on the propagation channel bandwidth size and transmitter and receiver antenna characteristics. In (8), $n$ and $k$ integers refer to relative delay and time sampling processing, respectively, with a sampling rate fixed to $F_{\text {sig. }}$. The model dynamically adapts the sampling rate of measurements to the simulated PHY layer system with a $2 \times 1 \mathrm{D}$ filtering optimization considering successive conversion rates in relative delay $\tau$ and time variation $t$ domains, respectively.

The conversion rate $R_{\mathrm{c}}$ by a non-integer factor, when passing from sampling rate $F_{1}$ to $F_{2}$, may be achieved by approximating $R_{c}$ as the ratio of two integers $L$ and $M$ (8) and use expansion and decimation operations combined with filter processing to remove $L-1$ duplicate forms of the interpolated signal and design low-band filter for decimation with a factor $M$ :

$$
R_{c}=\frac{F_{1}}{F_{2}} \approx \frac{L}{M}
$$

The CEPD model performs an optimization of multirate filter design to limit side lobes and preserve delay resolution during the conversion rate (CR) filter

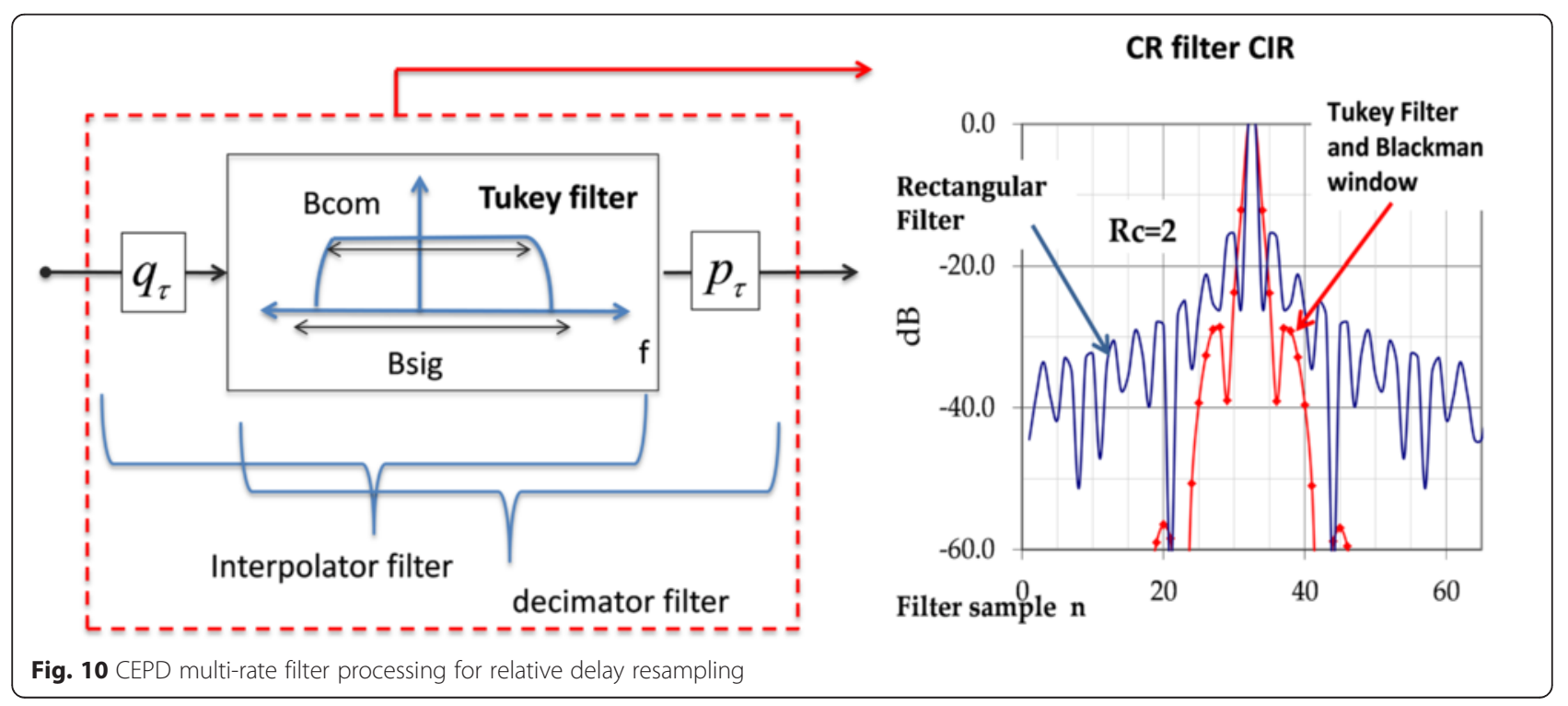




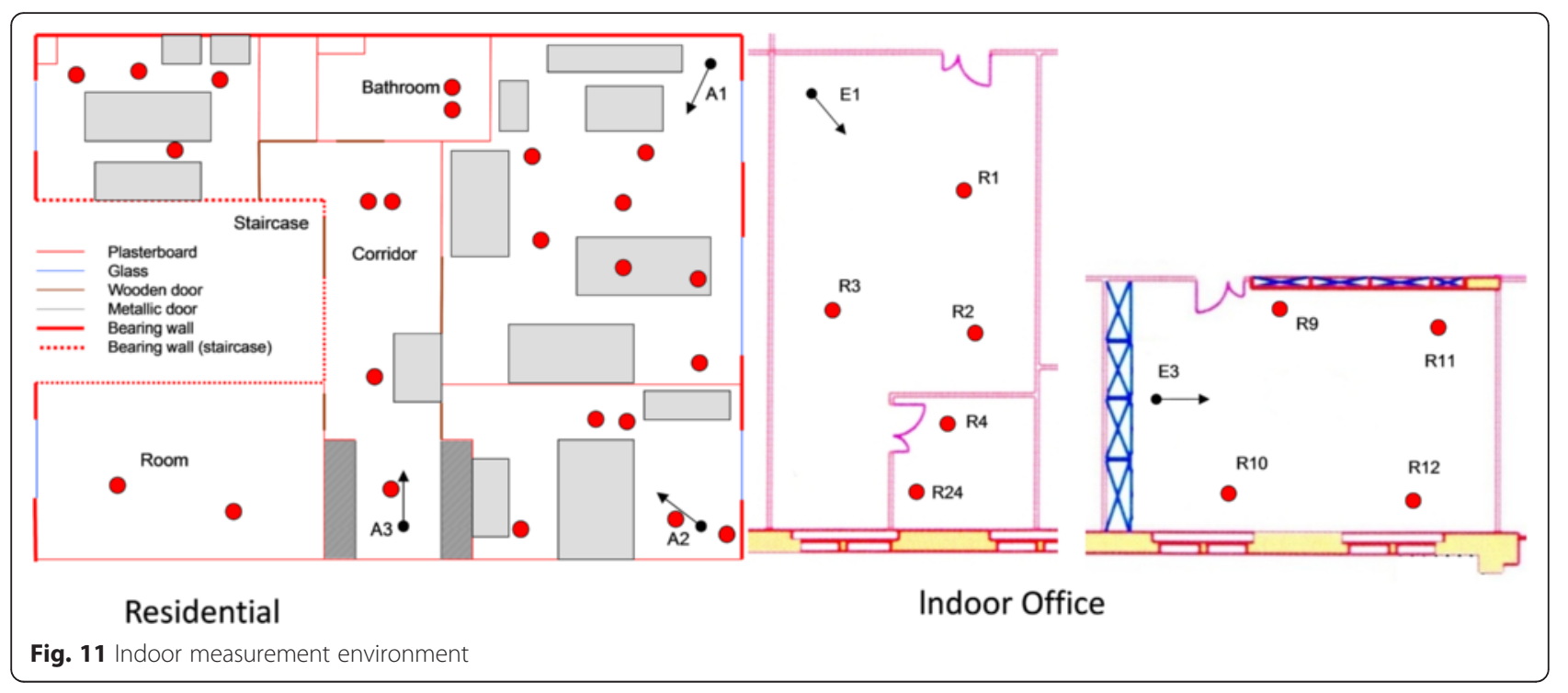

processing in the relative delay domain. Simple linear interpolation is done in the time domain $t$ to update the coefficients of the model. Assuming the PHY layer sampling rate is set to $F_{\text {sig }}$ and the propagation measurement sampling rate fixed to $F_{\text {init }}$, the relative delay $\tau$ conversion rate is then expressed as a ratio of two integers, $p$ and $q$, combining a $q$-interpolator filter followed with $p$-decimator filter to generate the link levelfocused propagation channel model. Filter processing is merged in a single filter design resulting from a Tukey filter setup in the frequency domain combined with a time-limited windowing process using a Blackman window. The conversion rate processing and resulting $\mathrm{CR}$ impulse response of the interpolator decimator filter are represented in Fig. 10, showing that the combination of filtering and windowing significantly reduces side lobes involved by filtering. Results are compared to a rectangular filter combined with a rectangular delay windowing.

The link level-focused propagation model is fed with dedicated CIR measurements carried out in larger bandwidths than the system bandwidth, involving filtering and lower sampling rate processing to generate the adequate CIR model. This model can be adapted to outdoor millimeter-wave overlay networks as well as to indoor deployments. Input files of the model issued from measurements provide the appropriate coefficients of the model attached to the simulated scenario. The candidate input files of the CEPD model result from a statistical analysis of a large amount of deterministic measurements, performed in each considered scenario. These scenarios are split into typical and atypical test cases including different levels of multipath dispersion. A typical test case in a deployment scenario results from CIR input files describing the average and median multipath dispersion of multipath selectivity parameters as the delay spread, the coherence bandwidth, the delay window set to $75 \%$, and the interval delay set to $6 \mathrm{~dB}$, while atypical cases are representative of severe situations corresponding to $90 \%$ of cumulative distribution function (CDF) of those selectivity parameters.

Assuming the first-order statistics of selectivity parameters follow a Gaussian distribution, the typical and atypical input files of the model are deduced from the CDFs of first-order statistic values of those parameters evaluated on $N$ moving experimental points composed each of $M$

Table 5 CEPD indoor deployment scenario

\begin{tabular}{lllll}
\hline Scenario & Antenna gain (dBi), Tx-Rx & Link & Deployment scenario & \\
\hline CM1 & $8-13$ & LOS & Antenna alignment & $\begin{array}{l}\text { Residential typical home with multiple rooms. } \\
\text { CM2 }\end{array} 8^{-13}$ \\
CM'1 & $8-24.6$ & NLOS/OLOS & No antenna alignment & \\
CM'2 & $8-24.6$ & LOS & Antenna mismatch alignment & \\
CM3 & $8-13$ & OLOS & & \\
CM4 & $8-13$ & LOS & Office antenna alignment & Office with typical office setup furnished with \\
multiple chairs, desks, computers, and workstations
\end{tabular}


Table 6 CEPD, selectivity parameters

\begin{tabular}{lcrrrrr}
\hline & $C M 1$ & \multicolumn{1}{c}{$C M_{2}$} & \multicolumn{1}{c}{$\mathrm{CM}^{\prime} 1$} & $\mathrm{CM}^{\prime} 2$ & $\mathrm{CM} 3$ & $\mathrm{CM} 4$ \\
\hline$\sigma_{\mathrm{DS}}(\mathrm{ns})$ & 2.36 & 6.94 & 9.41 & 9.41 & 6.37 & 7.11 \\
$\mathrm{BC}_{-0.5}(\mathrm{MHz})$ & 114 & 62.93 & 88.68 & 88.68 & 59.8 & 59.8 \\
\hline
\end{tabular}

static experimental measurement along a transmitterreceiver path. The CDFs of each selectivity parameter average are established for all measurement points and positions. The selected measurement point is selected if the average value of the concerned selectivity parameter is in the range of interval $I$ given by the Gaussian distribution.

The CDF is expressed as follows:

$$
\begin{aligned}
& \operatorname{Prob}\left\{\frac{|m i, j-X j|}{\sigma_{j}}<\alpha\right\}=\beta, \alpha=[10 \%-15 \%] \\
& I_{j}=\left\{X j-\alpha \cdot \sigma_{j}, X j+\alpha \cdot \sigma_{j}\right\}
\end{aligned}
$$

In eq. (9), $m_{i, j}$ is the average value of the selectivity parameter $j$ (RMS delay spread, coherence bandwidth, etc.) of the measurement point $i$ among $N . X_{j}$ and $s_{j}$ are the average and standard deviation, respectively, of the average selectivity parameter $j$ evaluated on $N$ points. The selected measurement point, i.e., the CIRs input file of the model, has the first order of selectivity parameter $j, m_{i, j}$, ranged in the $I_{j}$ interval. The procedure is iterated for all selectivity parameters indexed by integer $j$ in (9), leading to a selection of a restricted number of measurement points.

This section focuses on indoor multi-cluster modeling using indoor measurements carried out in a house (residential), with a single floor with a maximum transmitter $(\mathrm{Tx})$ to receiver $(\mathrm{Rx})$ distance of $12 \mathrm{~m}$, and in an office environment composed of several furnished desk rooms along a corridor of $20 \mathrm{~m}$.

NLOS corresponds to a transmitter and receiver not located in the same room. Transmissions are then performed by reflections on obstacles without alignment of transmitter and receiver antennas. OLOS refers to the transmitter and receiver located in the same room with partial obstructions. A map of measurements is given in Fig. 11, where several transmitter antenna positions are represented with black dots and measurement points with red dots in both residential and indoor office environments.

The channel sounding technique is based on a frequency sweep mode with a total bandwidth set to 1024 MHz using a VNA “AB millimètre 8-350" [30]. The VNA equipment presents a dynamic range of $40 \mathrm{~dB}$, and the channel is sampled at a rate of $0.1 \mathrm{~Hz}$. The channel transfer functions (CTFs) of the propagation channel have been measured in a frequency sweep mode with a total frequency bandwidth of $1024 \mathrm{MHz}$ and a frequency sweeping step fixed to $4 \mathrm{MHz}$, leading to an excess relative delay $\tau_{\max }$ of 250 ns. For each Tx-Rx configuration, the measured CTF was calibrated using a reference measurement in which the $\mathrm{Tx}$ and $\mathrm{Rx}$ ports of the sounder were directly cable connected. The corresponding CIRs have been obtained using an inverse Fourier transform combined with a Hanning window in order to reduce the level of secondary lobes in the relative delay domain due to the limited analyzed bandwidth.

Table 5 summarizes the propagation scenarios associated to measurements and antenna characteristics. At the transmitter side, a vertically polarized horn antenna

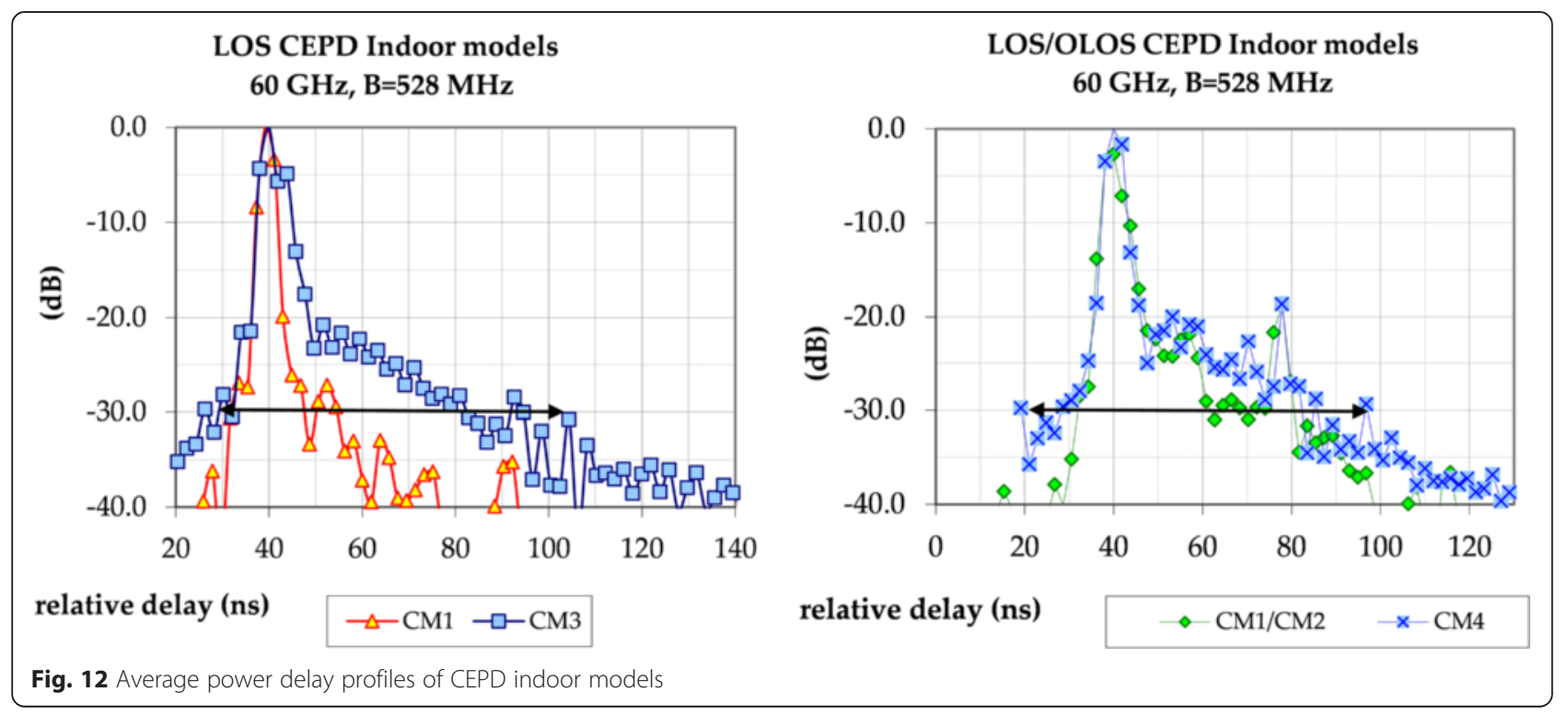


with a $72^{\circ}$ azimuth plane beam width and a gain of $8 \mathrm{dBi}$ was used. At the $\mathrm{Rx}$ positions, a vertically polarized narrow beam horn antenna $\left(10^{\circ}\right.$ azimuth plane beam width, 24.6-dBi gain) and a vertically polarized sectoral horn antenna $\left(60^{\circ}\right.$ azimuth plane pattern beam width, 13-dBi gain) were used in order to analyze antenna radiation pattern effects on multipath propagation characteristics. LOS and NLOS are discriminated. In Table 5, CM1 and CM2 scenarios refer to LOS and NLOS, respectively, in a residential environment with a correct antenna alignment between the transmitter and receiver sides. CM'1 and CM'2 are related to antenna alignment mismatch ranging from $2^{\circ}$ to $35^{\circ}$ with respect with antennas in alignment (LOS direction, $0^{\circ}$ ) in the same environment. CM3 and CM4 are complementary scenarios resulting from measurements carried out in an office environment in LOS and NLOS situations, respectively. No antenna alignment mismatch is considered in this scenario.

Selectivity parameters (RMS delay spread $\sigma_{\mathrm{DS}}$ and coherence bandwidth $B \mathrm{c}_{-0.5}$ ) given in eq. (10) of CEPD model realizations are detailed in Table 6. The RMS delay spread $\sigma_{\mathrm{DS}}$ is the average standard deviation of multipath echoes weighted by the power probability $\gamma_{I}$ of each relative delay $i$. The coherence bandwidth $B \mathrm{c}_{-0.5}$ is the frequency spacing for positive frequency components, providing a $1 / 2$ factor decrease of the normalized average correlation function $|\mathrm{RH}(\Delta f)|$ magnitude of the channel with respect to no frequency deviation $(\Delta f=0)$. In other words, the correlation coefficient adjusted to $1 / 2$ and $B c_{-0.5}$ represents the associated half-bandwidth size as expressed below:

$$
\begin{aligned}
& \gamma_{i}=E\left\{\frac{\mid h\left(t,\left.\tau_{i}(t)\right|^{2}\right.}{P(t)}\right\}, \\
& P(t)=\sum_{i=1}^{N}\left|h\left(t, \tau_{i}\right)\right|^{2}, \sigma_{D S}
\end{aligned}
$$

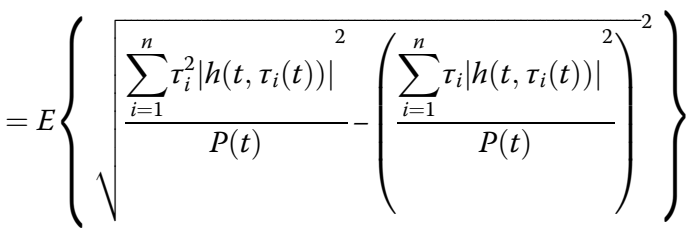

$$
\begin{aligned}
& \left.R H(\Delta f)=E\left\{F F T_{\tau}\left\{\sum_{i=1}^{n}\left|h\left(t, \tau_{i}(t)\right)\right|\right)^{2}\right\}(\Delta f)\right\}, \\
& B c-0.5=\Delta f^{\prime} \Rightarrow R H\left(\Delta f^{\prime}\right)=\frac{1}{2}|R H(\Delta f=0)|, \Delta f>0
\end{aligned}
$$

The average power delay profiles of indoor models related to indoor deployment scenarios (Table 5) are illustrated in Fig. 12.

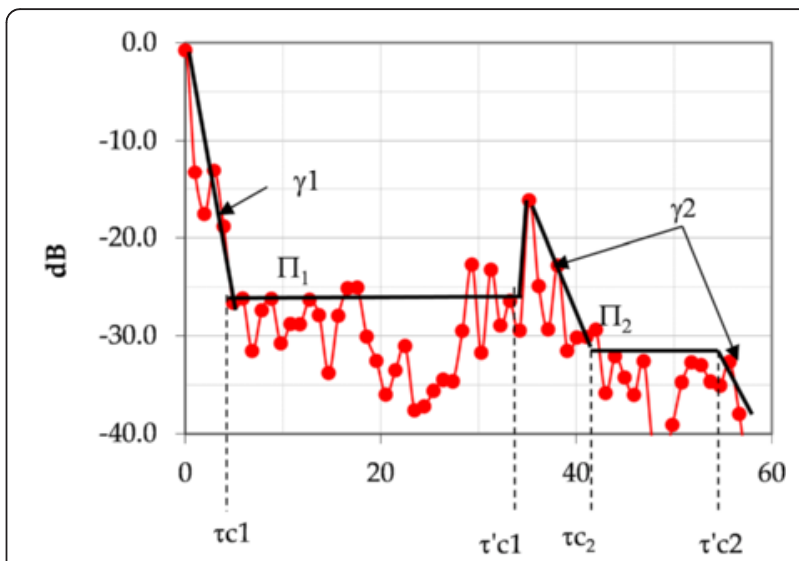

relative delay (ns)

Fig. 13 Average power delay profile of the CEPD model with an antenna alignment mismatch model, typical case, CM'1

CEPD antenna alignment mismatch models are derived from dedicated measurements followed by a CEPD selection procedure and multi-rate filter processing. Additionally, analytical models are designed that are derived from the multi-slope model [29] with an extension to a multi-cluster approach. The proposed model extends the concept to two clusters for each APDP deduced from CEPD realizations, encompassing two slope decays for each intra-cluster $\left(\gamma_{1}, y_{2}\right)$, two intra-cluster arrival time Poisson parameters $\left(\lambda_{1}, \lambda_{2}\right)$, two interval delays $(\Delta \tau I, \quad i=\{1,2\})$, and two constant multipath levels $(\Pi i(\Delta \tau i), i=\{1,2\})$ as illustrated in Figs. 13 and 14. The coefficients of the model given in Table 7 are deduced from CEPD realizations with a bandwidth of $528 \mathrm{MHz}$.

These analytical models derived from the CEPD realizations are an extension of the multi-slope model used to quantify antenna alignment mismatch. These models

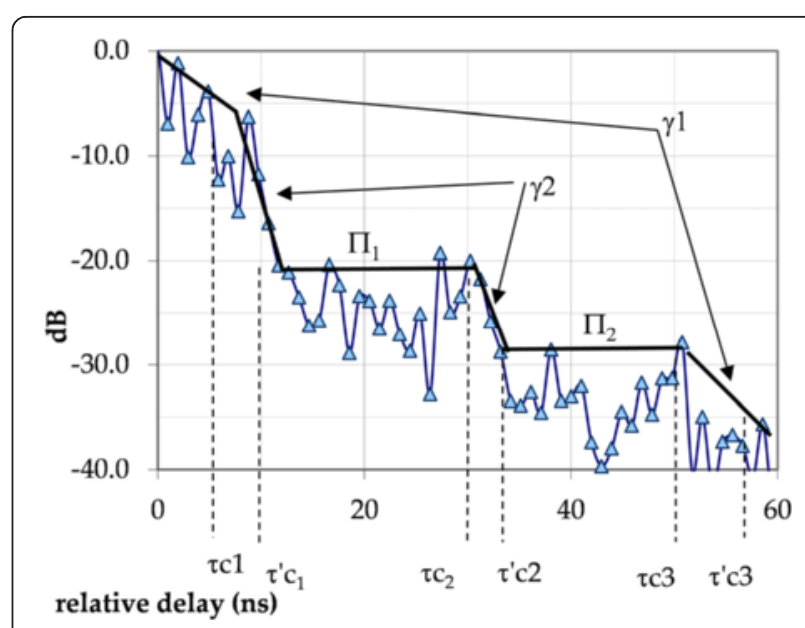

Fig. 14 Average power delay profile of the CEPD antenna alignment mismatch model, atypical case, CM'2 
Table 7 CEPD antenna alignment model coefficients

\begin{tabular}{|c|c|c|c|c|c|}
\hline & OLOS & OLOS & Delay & OLOS & OLOS \\
\hline & Typical & Atypical & & Typical & Atypical \\
\hline$\Pi 1(\mathrm{~dB})$ & -25 & -20.5 & $T C 1$ & 0.98 & 8.79 \\
\hline$\Pi 2(\mathrm{~dB})$ & -32.7 & -28.8 & $\tau^{\prime} \mathrm{C} 1$ & 35.19 & 11.71 \\
\hline$\gamma_{2}(\mathrm{~dB} / \mathrm{ns})$ & 1.81 & 4.86 & TC2 & 43.97 & 30.27 \\
\hline$\lambda_{2}$ (1/ns) & 0.42 & 1.12 & $t^{\prime} \mathrm{C} 2$ & 55.69 & 33.2 \\
\hline$\gamma_{1}(\mathrm{~dB} / \mathrm{ns})$ & 4.45 & 0.72 & TC3 & & 50.78 \\
\hline$\lambda 1(1 / \mathrm{ns})$ & 1.02 & 0.17 & $t^{\prime} \mathrm{c} 3$ & & 58.59 \\
\hline$d(T x-R x)$ & 5.84 & 735 & & & \\
\hline
\end{tabular}

and associated CEPD realizations may be used to evaluate involved degradations on link level performance and highlight benefits of fast-tracking beam-forming in time variant environments [31].

\section{Conclusions}

A Q-D channel model and a link level-focused model were introduced. The two models follow different approaches. While the Q-D model gives a full understanding of the spatial channel, it also requires a precise description of the scenario. This spatial resolution, as well as the spatial consistency of the model for moving users, is of key importance for link level design. With the expected shift towards steerable antennas with medium or high gain, the antenna pattern has a much higher influence on the effective channel between the transmitter and receiver. Aspects like initial discovery of millimeter-wave base stations and beacon design also heavily depend on the spatial information.

The flexibility of this Q-D approach allows channel models for access links to be used for scenarios with similar geometries. For example, the "street canyon access mode" can be changed to the "street-level backhaul model" by changing the receiver antenna parameters. These models were validated with different measurements in outdoor scenarios. The Q-D open area channel model was successfully applied to millimeter-wave multi-user MIMO (MU MIMO) small cell access link scenario evaluations [32-34]. Future work remains on some aspects of the model, like the modeling of the flashing rays (F-rays) and NLOS scenarios, where further measurement campaigns are necessary.

The CEPD model results from an optimized multi-rate filtering combined with a statistical analysis of measurements. It allows an extraction of typical (average behavior of the multipath channel related to a measurement campaign) and atypical (severe cases representative of measurement campaign) measurements that are used as inputs of the model to generate model coefficients associated to the targeted scenario. The model is dedicated to link level simulations with multiple underlying technologies and bandwidths. Scenarios covered in the paper consider antenna alignment mismatch where CEPD realizations have been exploited to generate analytical multi-cluster models derived from the multi-slope concept. The CEPD model can also use the Q-D model as an input to the interface with link level simulations. When the link level is not the focus of research, this is a convenient method of abstraction in order to reduce the complexity for larger scale simulations, for example, with multiple users and multiple base stations.

Competing interests

The authors declare that they have no competing interests.

\section{Acknowledgements}

The results presented are from the European part of MiWEBA which is a STREP-funded project by the European Union under grant agreement no. 608637 as part of FP7 ICT-2013-EU-Japan [11]. This work was funded with the support from the EU FP7 program, and the results are also publicly available on the project website http://www.miweba.eu.

Furthermore, the authors would like to acknowledge the support of their colleagues, MiWEBA project partners, and the COST IC1004 for their support and fruitful discussions.

\section{Author details}

${ }^{1}$ Fraunhofer Heinrich-Hertz-Institute, Berlin, Germany. ${ }^{2}$ Platform Engineering Group, Intel Corporation, Santa Clara, USA. ${ }^{3}$ University of Nizhny Novgorod, Nizhny Novgorod, Russia. ${ }^{4}$ Orange Labs Cesson, Cesson Sevigne, France.

Received: 31 July 2015 Accepted: 24 February 2016

Published online: 15 March 2016

References

1. F Boccardi, RW Heath, A Lozano, TL Marzetta, P Popovski, Five disruptive technology directions for 5G. IEEE Communications Magazine 52(2), 74-80 (2014)

2. ETSI Group Specification mWT 002, "Millimetre wave transmission (mWT); applications and use cases of millimetre wave transmission," V1.1.1 (2015-08), 2015.

3. TS Rappaport, S Sun, R Mayzus, H Zhao, Y Azar, K Wang, GN Wong, JK Schulz, M Samimi, F Gutierrez, Millimeter wave mobile communications for $5 \mathrm{G}$ cellular: it will work! IEEE Access 1, 335-349 (2013)

4. M. Coldrey (ed.) "Maturity and field proven experience of millimetre wave transmission," ETSI White Paper No. 10, First edition-September 2015, ISBN 9791092620078, 2015

5. P. Kyösti, J. Meinilä, L. Hentilä, X. Zhao, T. Jämsä, C. Schneider, M. Narandzić, M. Milojević, A. Hong, J. Ylitalo, V.-M. Holappa, M. Alatossava, R. Bultitude, Y. de Jong, T. Rautiainen, "WINNER II channel models", tech. rep. D1.1.2 V1.2, IST-4-027756 WINNER II, 2007

6. "Channel models for $60 \mathrm{GHz}$ WLAN systems," IEEE document 802.11-09/0334r8

7. A Maltsev, AR Maslennikov, AK Sevastyanov, A Lomayev, Experimental investigation of $60 \mathrm{GHz}$ wireless systems in office environment. IEEE JSAC 27(8), 1488-1499 (2009)

8. A Maltsev, A Pudeyev, I Karls, I Bolotin, G Morozov, RJ Weiler, M Peter, W Keusgen, Quasi-deterministic approach to $\mathrm{mm}$-wave channel modeling in a non-stationary environment (IEEE GLOBECOM, Austin, 2014)

9. A. Maltsev, A. Pudeyev, I. Karls, I. Bolotin, G. Morozov, R.J. Weiler, M. Peter, W. Keusgen, M. Danchenko, A. Kuznetsov, "Quasi-deterministic approach to Mm-wave channel modeling in the FP7 MiWEBA project," WWRF' 33 (Wireless World Research Forum (WWRF), Guildford, GB, 2014)

10. I. Siaud, A.-M. Ulmer-Moll, A. Capone, A. Filippini, A. De Domenico, E. Calvanese-Strinati, R. Weiler, K. Sakaguchi, "Definition of Scenarios and Use Cases", tech. rep. D1.1, v1.0, FP7-ICT-608637 MiWEBA, 2013

11. "MiWEBA project homepage http://www.miweba.eu (FP7-ICT-2013-EU-Japan, project number: 608637)," 2015

12. M. Peter, W. Keusgen and R.J. Weiler, "On path loss measurement and modeling for millimeter-wave 5G," in EUCAP 2015. 
13. R. J. Weiler, M. Peter, W. Keusgen and M. Wisotzki, "Measuring the busy urban $60 \mathrm{GHz}$ outdoor access radio channel," in ICUWB, 2014

14. R.J. Weiler, M. Peter, T. Kühne, M. Wisotzki, W. Keusgen, "Simultaneous millimeter-wave multi-band channel sounding in an urban access scenario," in EUCAP, 2015

15. ICT FP7 MiWEBA project \#608637, 'Deliverable D5.1, channel modeling and characterization', Public deliverable, Intel editor, June 2014.

16. RJ Weiler, M Peter, W Keusgen, A Kortke, M Wisotzki, Millimeter-wave channel sounding of outdoor ground reflections (IEEE Radio and Wireless Symposium (RWS), San Diego, 2015)

17. R. J. Weiler, M. Peter, W. Keusgen, H. Shimodaira, K. T. Gia and K. Sakaguchi, "Outdoor millimeter-wave access for heterogeneous networks-path loss and system performance," in PIMRC, 2014

18. A Hammoudeh, M Sanchez, E Grindrod, Modelling of propagation in outdoor microcells at 62.4GHz. Microwave Conference 1, 119-123 (1997)

19. K Sarabandi, E Li, A Nashashibi, Modeling and measurements of scattering from road surfaces at millimeter-wave frequencies. IEEE Transactions on Antennas and Propagation 45(11), 1679-1688 (1997)

20. A Yamamoto, K Ogawa, T Horimatsu, A Kato, M Fujise, Path-loss prediction models for intervehicle communication at $60 \mathrm{GHz}$. IEEE Transactions on vehicular technology 57, 1 (2008)

21. ITU Report 1008-1, Reflection from the surface of the Earth

22. A Maltsev, E Perahia, R Maslennikov, A Sevastyanov, A Lomayev, A Khoryaev, Impact of polarization characteristics on $60-\mathrm{GHz}$ indoor radio communication systems. IEEE Antennas And Wireless Propagation Letters 9, 413 (2010)

23. Sawada H., "Intra-cluster response model and parameter for channel modeling at $60 \mathrm{GHz}$ (Part 3)", IEEE doc. 802.11-10/0112r1, January 2010.

24. IMST $60 \mathrm{GHz}$ indoor radio channel measurement data, ACTS MEDIAN project technical report.

25. K Sato, T Manabe, T Ihara, H Saito, S Ito, T Tanaka, K Sugai, N Ohmi, Y Murakami, M Shibayama, Y Konishi, T Kimura, Measurements of reflection and transmission characteristics of interior structures of office building in the 60-GHz band. Antennas and Propagation, IEEE Transactions on 45(12), 1783-1792 (1997)

26. Davydov A., Maltsev A., Sadri A., "Saleh-Valenzuela channel model parameters for library environment," IEEE document 802.15-06-0302-02-003c, July 2006

27. R. E. Crochiere, L.R. Rabiner, Multi-rate digital signal processing, (Prentice-Hall, New Jersey, 1983), ISBN 0136051626

28. I. Siaud, A.M. Ulmer-Moll, N. Malhouroux-Gaffet, V. Guillet, Short-range wireless communications, an introduction to $60 \mathrm{GHz}$ communication systems: regulation and services, channel propagation and advanced baseband algorithms, Chapter 18, ed. Rolf Kraemer and Marcos D. Katz (The Atrium, Southern Gate, Chichester, West Sussex, PO19 8SQ, United Kingdom, John Wiley \& Sons Ed. 2009). ISBN: 978-0-470-69995-9

29. H. Yang, F.M. Smulders \& Matti H.A.J, Herben, Channel characteristics and transmission performance for various channel configurations at $60 \mathrm{GHz}$. Eur. J. Wireless Commun. Netw.", 2007(1), 43-43 (2007), Hindawi Publishing Corp. New York, NY, United States, DOl10.1155/2007/19613.

30. P Goy, S Caroopen, M Gross, Vector measurements at millimeter and submillimeter wavelengths: feasibility and applications (ESA Workshop on Millimeter Wave Technology and Applications, Espoo, 1998)

31. I Siaud, AM Ulmer-Moll, N Cassiau, MA Bouziques, Adaptive and spatial processing for millimeter wave backhaul architectures (International Conference ICUWB', Montreal, 2015)

32. A. Maltsev, A. Sadri, A. Pudeyev, R. Nicholls, R. Arefi, A. Davydov, I. Bolotin, G. Morozov, K. Sakaguchi and T. Haustein, "MmWave small cells is a key technology for future $5 \mathrm{G}$ wireless communication systems," in European Conference on Networks and Communications, 2014

33. A. Maltsev, A. Sadri, A. Pudeyev, A. Davydov, I. Bolotin, G. Morozov, "Performance evaluation of the MmWave small cells communication system in MU-MIMO mode", EuCNC'2015.

34. ICT FP7 MiWEBA project 608637, 'Deliverable D4.1, system level simulator specification', Public deliverable, http://www.miweba.eu/wp-content/ uploads/2014/07/MiWEBA_D4-1_v10.pdf

\section{Submit your manuscript to a SpringerOpen ${ }^{\circ}$ journal and benefit from:}

- Convenient online submission

- Rigorous peer review

- Immediate publication on acceptance

- Open access: articles freely available online

- High visibility within the field

- Retaining the copyright to your article

Submit your next manuscript at $\gg$ springeropen.com 